\title{
Seed Health Evaluation of Pea Varieties by Incubation Methods
}

\author{
Ashruti Kesharwani*, N. Lakpale, N. Khare and P.K. Tiwari \\ Department of Plant Pathology, IGKV, Raipur (CG) 490 012, India \\ *Corresponding author
}

\section{A B S T R A C T}

Keywords

Pea, Seed borne mycoflora, Seed health

\section{Article Info}

\section{Accepted:}

06 July 2018

Available Online:

10 August 2018
Like other crops, pea varieties were also harbour the seed borne mycoflora resulted in less germination and plant vigour index. Six varieties of pea and one local variety were taken in the present investigation to assess the seed health by incubation methods. It was found that varying degree and type of mycoflora associated with pea seeds were recorded. The mycoflora recorded were Aspergillus niger, Aspergillus flavus, Penicillium sp., Trichoderma sp., Basidiophora sp., Chaetomium sp., Aspergillus fumigatus, Alternaria sp., Curvularia sp., Fusarium sp., Rhizopus sp. and Rhizoctonia sp. In all these methods, poor germination was observed in local variety, may be due to presence of seed borne mycoflora with higher frequencies whereas Shubhra variety has higher germination due to lower frequencies of detected mycoflora as compared to other varieties of pea taken in the study.

\section{Introduction}

Pea is the third most important pulse crop at global level, after dry bean and chickpea and third most popular Rabi pulse of India after chickpea and lentil. It is originated from Mediterranean Region of Southern Europe and Western Asia. It provides a variety of vegetarian diet hence liked throughout the world. The mature seeds are used as whole or split into dal and put to use in various ways for human consumption. Beside vegetable purposes, it is also grown as a forage crop for cattle and cover crop to prevent soil erosion but mainly for matured seed for human consumption. The seeds are highly nutritious as it contains about $22.5 \%$ protein, 64 $\mathrm{mg} / 100 \mathrm{~g}$ calcium, $1.8 \%$ fat, $4.8 \mathrm{mg} / 100 \mathrm{~g}$ iron, $62.1 \%$ carbohydrate and $11 \%$ moisture.
Healthy seed is the foundation of healthy plant, a necessary condition for good yield (Diaz et al., 1998). Seed health is affected by various factors, among which the most important is seed borne fungi that cause reduction in seed germination and seed vigour. Germination, growth and productivity of crop plants are affected by the infection of various mycoflora. A seed-borne pathogen present externally or internally or associated with the seed as contaminant may cause seed abortion, seed rot, seed necrosis, reduction or elimination of germination capacity as well as seedling damage resulting in the development of disease at later stages of plant growth of systemic or local infection.

Ten different fungi namely Alternaria sp., Aspergillus flavus, A. niger, Chaetomium 
globosum, Fusarium oxysporum, Fusarium sp., Cladosporium cladosporioides, Rhizoctonia solani, Penicillium sp. and Curvularia lunata were associated with pea seeds (Khan et al., 2006).

Till date, seed health evaluation aspects like mycoflora associated, their effect on seedling vigour index, embryo infection, transmission and management not studied well and documented of different pea varieties of Chhattisgarh. Therefore, an attempt is taken to carry out the present investigation to assess the seed health of pea varieties by incubation methods.

\section{Materials and Methods}

Unless and otherwise mentioned for each experiment, 400 seeds were used. In general, the petridish with seeds were incubated at $22 \pm 1^{\circ} \mathrm{C}$ under a 12 hours' dark and light cycle with NUV light for seven days. Observations were recorded seven days after seeding for the type of mycoflora associated and its frequency. The organisms were observed under the stereobinocular microscope on seeds for their habit characters and confirmed under compound microscope.

The associated mycoflora were identified with the help of standard literature like Illustrated Genera of Imperfect Fungi (H.L. Barnett, 1962), More Dematiaceous Hypomycetes (M.B. Ellis, 1976) and A Pictorial Guide to the Identification of Seed borne Fungi of Sorghum, Pearl Millet, Finger Millet, Chickpea, Pigeonpea and Groundnut (ICRISAT, 1978).

Several methods of seed health testing have been developed for the detection of fungi associated with seeds. Some of the most suitable procedures have been recommended by the International Seed Testing Association (ISTA) (Anon., 1966). The methods to detect microbes in or on the seed vary quite markedly depending on the location of the microbes and the mode of seed transmission (Neergaard, 1977) and the particular group to which the microbe belongs.

The following standard methods recommended were used in the present study for seed health evaluation of different varieties of pea.

Standard blotter method (ISTA, 1976)

Agar plate method (Muskett and Malone, 1941)

Roll paper towel method (Yaklich, 1985)

Deep freeze method (Limonard, 1968)

\section{Standard blotter method}

This method was used to detect the presence of fungi on or in the seeds after incubation. By this method, the fast growing fungi were better detected than the slow growing ones. In each inter-fitting plastic petri plate, two good quality blotter papers of the same diameter were kept and moistened with sterilized distilled water.

In each plate, ten seeds were placed on the moistened blotters in such a manner that nine seeds formed the outer circle and one at the center. For each lot of seed, 40 replicated plates were maintained (total of 400 seeds tested for each lot of seed). Incubate the plate at $22 \pm 1^{\circ} \mathrm{C}$ for seven days in alternating cycles of 12 hours' darkness and 12 hours' light in NUV.

Observations were recorded as described earlier. All seeds of the outer ring were examined first, finally the seed in the centre of the dish and expressed in per cent seed mycoflora associated, individually. 
The frequency of the fungus was calculated by the following formula

No. of seeds containing a particular fungus

Total seeds examined

Total no. of colonies of a fungus on seeds Relative abundance $=\longrightarrow \times 100$

Total no. of colonies of all fungus

\section{Agar plate method}

Potato dextrose agar medium (15-20 ml) was poured in each sterilized petri plate. To avoid bacterial contamination, a little amount of Streptomycin sulphate was added in the medium at the time of pouring. Seeds of each lot were surface sterilized with $1.0 \% \mathrm{NaOCl}$ solution for 30 seconds and immediately washed twice with sterile distilled water thoroughly to remove $\mathrm{NaOCl}$ solution that adhered if any. Seeds were placed on the previously poured PDA medium in Petri plate in such a way that nine seeds in the outer circle and one at the centre. For each lot, 40 replicated plates were maintained and incubated at $22 \pm 1^{\circ} \mathrm{C}$ under alternate cycles of $12 \mathrm{hrs}$ dark and $12 \mathrm{hrs}$ light in NUV. Observations were recorded as described earlier.

\section{Roll paper towel method}

The seed were placed on moist paper towel (50-100) at equidistance and covered with another moist paper towel and rolled carefully without disturbing the already arranged seeds. Tie the towel with rubber band at both the ends. To avoid water loss, use wax coated paper or polythene rapping the rolled towels containing seeds. Incubate it for four to five days at room temperature. Examine normal and abnormal seedlings, ungerminated seeds, cause of abnormalities and failure in germination by naked eye and presence of mycoflora by stereo-binocular microscope. The observations were recorded for-

\section{Normal seedlings}

Show the potential for continued development into satisfactory plants when grown in good quality soil and under favorable conditions of moisture, temperature and light.

\section{Categories}

\section{Intact seedlings}

Seedlings with all their essential structures well developed, complete in proportion and healthy.

\section{Seedlings with slight defects}

Seedlings showing slight defects of their essential structures provided they show a satisfactory and balanced development comparable to that of intact seedlings of the same test.

\section{Seedlings with secondary infection}

Seedlings as described above but have been affected by fungi or bacteria from sources other than the parent seed.

\section{Abnormal seedlings}

Do not show the potential to develop into a normal plant when grown in good quality soil and under favorable condition of moisture, temperature and light.

\section{Categories}

\section{Damaged}

Seedlings with any of the essential structures missing or badly and irreparably damaged that balance development cannot be expected. 


\section{Deformed or unbalanced}

Seedlings with weak development or physiological disturbances or in which essential structures are deformed or out in proportion.

\section{Decayed}

Seedlings with any of their essential structures so diseased or decayed

As a result of primary infection that normal development is prevented.

\section{Ungerminated seeds}

Did not germinate at the end of the test period.

\section{Categories}

\section{Hard seeds}

Seeds which have not absorbed water thus remain. Hard after the end of the test period.

\section{Fresh seeds}

Seeds able to imbibe water but which failed to germinate under the condition of the germination test remain clean and firm and have the potential to develop into a normal seedling.

\section{Dead seeds}

Seeds at the end of the test period are neither hard nor fresh,

Failed to proof a seedling; usually soft, discolored, frequently moldy.

\section{Others}

Empty, embryo less seeds, insect damaged seeds.

\section{Deep freeze method}

It was a modification of standard blotter method. The seeds were plated on blotters moistened with a solution containing $0.2 \%$ Streptopenicillin (to avoid bacterial contamination) and incubated for 24 hours under normal conditions in growth chamber. Plates were further incubated at $10 \pm 1^{\mathrm{O}} \mathrm{C}$ for three days and then transferred to deep freezer $\left(-20^{\circ} \mathrm{C}\right)$ under complete darkness for 24 hours. Plated were again incubated at $20-25{ }^{\circ} \mathrm{C}$ for 57 days. Observations were recorded as described earlier.

\section{Results and Discussion}

\section{Standard blotter method}

Seed lots of different pea varieties were evaluated for the associated seed borne mycoflora by using standard blotter method and data presented in table 1 indicates that maximum frequency of mycoflora were recorded from the seed lot of local variety $(111.25 \%)$ with lowest germination percentage (75.76) and six mycoflora were detected as Aspergillus flavus (21\%), Aspergillus niger (10.50\%), Aspergillus fumigatus (6\%), Alternaria sp. (33.50\%), Chaetomium sp.(24.75\%) and Rhizopus sp. (15.5\%). This was followed by KPMR 400 seed lot $(97.50 \%)$ and five mycoflora were detected as Aspergillus flavus (29.25\%), Aspergillus niger (9.25\%), Trichoderma sp. (15.00\%), Alternaria sp. (39.25\%) and Chaetomium sp. (4.75\%) with germination percentage 83.50 .

In the seed lot of Ambika, Indira matar, IPFD 10-12 and Paras frequency of mycoflora associated were $95.50 \%, 87.75 \%, 80.75 \%$ and $77 \%$, respectively. In Shubhra variety minimum frequency of mycoflora $(65.5 \%)$ were recorded and mycoflora detected as Aspergillus flavus (15.5\%), Aspergillus niger 
(18.5\%), Aspergillus fumigatus (4.5\%) and Chaetomium sp. (27\%) with maximum germination percentage 98.25 .

The relative bundance of Aspergillus flavus $(158.5 \%)$ were found maximum from seed lots of pea varieties and it appeared frequently in Ambika (33.25\%) followed by Indira matar (31\%) and KPMR 400 (29.25\%). Other predominant mycoflora were Alternaria sp. (131.75\%), Chaetomium sp. (108.25\%), Aspergillus niger (66.75\%), Basidiophora sp. (39.75\%), Curvularia sp. (31.75\%), Aspergillus fumigatus (24.75\%), Rhizocpus sp. (24\%) and Trichoderma sp. (21.25\%). Penicillium sp. were detected with least frequency $(8.75 \%)$ and detected only in Paras variety.

In this method, local variety shows the highest frequency of mycoflora with lowest germination percentage while least frequency of mycoflora with highest germination percentage was recorded in Shubhra variety among all the varieties taken in the study. Pea varieties were found associated with Aspergillus flavus in high frequency and Penicillium sp. were found with least frequency.

Rauf (2000), Begum et al., (2004), Verma et al., (2004), Narayan et al., (2013) and Ghangaokar et al., (2013) also identified various seed borne mycoflora associated with pea in varying frequencies by blotter paper method. These finding are in agreement with the findings of present study, in which decreasing trend in seed germination and increasing trend in seed mycoflora were recorded. In other legumes also, standard blotter method appeared as useful in detecting seed borne mycoflora efficiently. Barua et al., (2007), Ashwini et al., (2014), Mohana et al., (2015) and Pradhan (2017) reported in green gram; Dawar et al., (2007), Ghangaokar et al., (2013), Margaret et al., (2013), Sontake et al.,
(2014), Mohana et al., (2015), Kumar (2016) reported in chickpea and Patil et al., (2012), Ghangaokar et al., (2013), Pradhan (2014) and Chaudhary et al., (2017) reported in pigeonpea

\section{Agar plate method}

Seeds of seven pea varieties were evaluated for associated seed borne mycoflora in agar plate method and data presented in table 2 . Maximum frequency of mycoflora were recorded from seeds of local variety (131.25\%) which include Aspergillus flavus (53.5\%), Aspergillus niger (28.75\%), Basidiophora sp. (5\%), Rhizopus sp. (10.75\%), Rhizoctonia sp. (14.25\%) and Fusarium sp. (19\%) with least germination $(58.5 \%)$ followed by KPMR 400 seed lot $(113 \%)$ with germination percentage 69.25. Frequencies of mycoflora recorded from the seeds of other pea varieties were Ambika (103.65\%), IPFD10-12 (102.50\%), Paras $(97 \%)$ and Indira matar $(81.75 \%)$ with varying germination percentage as $83.25,84.5,89.25$, and 94, respectively. Least frequency of mycoflora were recorded from seeds of Shubhra variety $(80 \%)$ with highest germination percentage -97.75 .

Various mycoflora were detected from pea varieties by this method, in which relative abundance of Aspergillus flavus (250.5\%) was recorded and it was most frequent in KPMR $400(62.75 \%)$ and local variety $(53.5 \%)$. This was followed by Rhizoctonia sp. (112.4\%), Rhizopus sp. (94.5\%), Aspergillus niger (69.75\%), Fusarium sp. (60\%), Trichoderma sp. $(44.25 \%)$ and Aspergillus fumigatus (40.25\%). Basidiophora sp. (37.5\%) were found with least frequency in pea varieties.

It appears that among all pea varieties, local variety showed maximum frequency of mycoflora and lowest germination, while Shubhra variety showed minimum frequency of mycoflora with maximum germination. 
Table.1 Detection of mycoflora associated with seeds of pea by Standard Blotter Method

\begin{tabular}{|c|c|c|c|c|c|c|c|c|c|c|c|c|}
\hline \multirow[b]{2}{*}{ Varieties } & \multirow[b]{2}{*}{$\begin{array}{l}\text { Germina } \\
\text { tion } \\
(\%)\end{array}$} & \multicolumn{10}{|c|}{ Frequency of mycoflora associated (\%) } & \multirow{2}{*}{$\begin{array}{l}\text { Total } \\
\text { freque } \\
\text { ncy } \\
(\%)\end{array}$} \\
\hline & & \multirow[t]{2}{*}{ క్ర } & \multirow{2}{*}{ 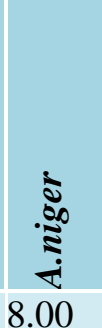 } & \multirow[t]{2}{*}{ 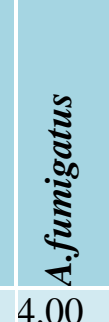 } & \multirow[t]{2}{*}{ 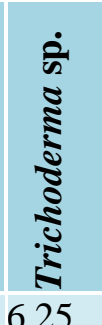 } & \multirow[t]{2}{*}{ 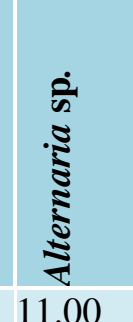 } & \multirow[t]{2}{*}{ 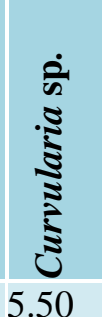 } & \multirow[t]{2}{*}{ 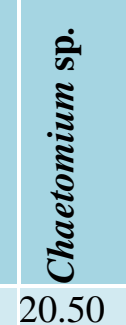 } & \multirow[t]{2}{*}{ 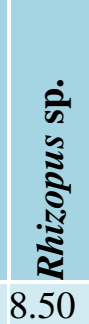 } & \multirow[t]{2}{*}{ 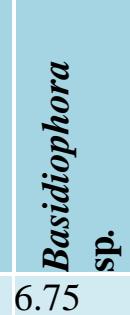 } & 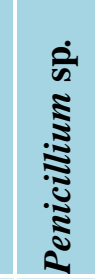 & \\
\hline IPFD10-12 & 90.50 & & & & & & & & & & - & 80.75 \\
\hline Paras & 97.50 & 18.25 & 7.75 & 6.50 & - & 12.00 & 6.75 & 7.50 & - & 9.50 & 8.75 & 77.00 \\
\hline KPMR 400 & 84.50 & 29.25 & 9.25 & - & 15.00 & 39.25 & - & 4.75 & - & - & - & 97.50 \\
\hline Indira matar & 85.00 & 31.00 & - & 3.75 & - & 22.50 & 19.5 & - & - & 11.00 & - & 87.75 \\
\hline Shubhra & 98.25 & 15.50 & 18.50 & 4.50 & - & - & - & 27.00 & - & - & - & 65.50 \\
\hline Ambika & 83.50 & 33.25 & 12.75 & - & - & 13.5 & - & 23.75 & - & 12.25 & - & 95.50 \\
\hline Local variety & 75.76 & 21.00 & 10.50 & 6.00 & - & 33.50 & - & 24.75 & 15.5 & - & - & 111.25 \\
\hline \multicolumn{2}{|c|}{ Total mycoflora } & 158.50 & 66.75 & 24.75 & 21.25 & 131.75 & 31.75 & 108.25 & 24 & 39.75 & 8.75 & \\
\hline
\end{tabular}

Table.2 Detection of mycoflora associated with seeds of pea by agar plate method

\begin{tabular}{|c|c|c|c|c|c|c|c|c|c|c|}
\hline \multirow[t]{2}{*}{ Varieties } & \multirow{2}{*}{$\begin{array}{l}\text { Germina } \\
\text { tion }(\%)\end{array}$} & \multicolumn{8}{|c|}{ Frequency of mycoflora associated (\%) } & \multirow{2}{*}{$\begin{array}{l}\text { Total } \\
\text { frequency }\end{array}$} \\
\hline & & 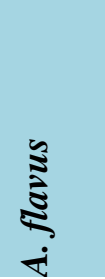 & 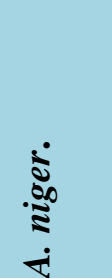 & 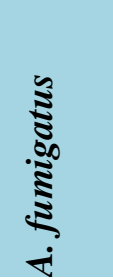 & 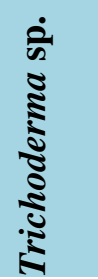 & 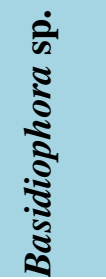 & 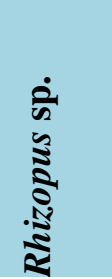 & 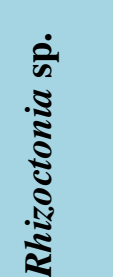 & 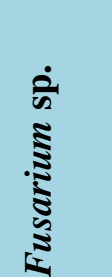 & \\
\hline $\begin{array}{l}\text { IPFD 10- } \\
12\end{array}$ & 84.50 & 48.25 & - & 5.00 & - & 13.75 & 35.50 & - & - & 102.50 \\
\hline Paras & 89.25 & 19.00 & 14.75 & 6.75 & - & 7.00 & 11.00 & 25.75 & 12.75 & 97.00 \\
\hline $\begin{array}{l}\text { KPMR } \\
400\end{array}$ & 69.25 & 62.75 & - & 28.50 & - & 11.75 & - & 10.00 & - & 113.00 \\
\hline $\begin{array}{l}\text { Indira } \\
\text { Matar }\end{array}$ & 94.00 & 33.25 & 5.25 & - & 6.25 & - & 12.25 & 13.25 & 11.50 & 81.75 \\
\hline Shubhra & 97.75 & 18.75 & 10.00 & - & 26.25 & - & 25.00 & - & - & 80.00 \\
\hline Ambika & 83.25 & 15.00 & 11.00 & - & 11.75 & - & - & 49.15 & 16.75 & 103.65 \\
\hline $\begin{array}{l}\text { Local } \\
\text { Variety }\end{array}$ & 58.50 & 53.50 & 28.75 & - & - & 5.00 & 10.75 & 14.25 & 19.00 & 131.25 \\
\hline \multicolumn{2}{|c|}{ Total mycoflora } & 250.5 & 69.75 & 40.25 & 44.25 & 37.50 & 94.50 & 112.4 & 60.00 & \\
\hline
\end{tabular}


Table.3 (a) Detection of mycoflora associated with seeds of pea by roll paper towel method

\begin{tabular}{|c|c|c|c|c|c|c|c|c|c|c|}
\hline \multirow[t]{2}{*}{ Varieties } & \multirow{2}{*}{$\begin{array}{l}\text { Germination } \\
(\%)\end{array}$} & \multicolumn{8}{|c|}{ Frequency of mycoflora associated (\%) } & \multirow{2}{*}{$\begin{array}{l}\text { Total } \\
\text { Frequency }\end{array}$} \\
\hline & & $\dot{\tilde{\Sigma}}$ & 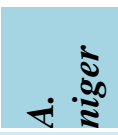 & 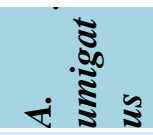 & $\frac{\sqrt{5}}{\sqrt[3]{5}} \frac{\pi}{3}$ & 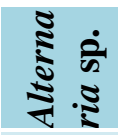 & 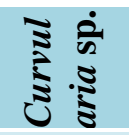 & 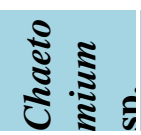 & 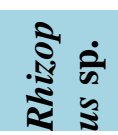 & \\
\hline IPFD 10-20 & 93.98 & 11.66 & 5.33 & 6.66 & 1.66 & 37.00 & 15.33 & - & 1.66 & 79.30 \\
\hline Paras & 96.98 & 19.66 & 6.33 & - & 8.00 & - & 17.00 & 11.66 & 12.00 & 74.65 \\
\hline KPMIR 400 & 92.65 & 20.00 & 12.66 & 1.33 & 4.33 & 20.00 & 12.66 & 5.33 & 6.66 & 82.97 \\
\hline Indira matar & 94.32 & 11.33 & 15.00 & 4.66 & 5.33 & 28.00 & 7.66 & 6.33 & - & 78.31 \\
\hline Shubhra & 98.98 & 17.33 & 3.66 & - & 7.00 & - & 32.00 & 3.66 & 3.00 & 66.65 \\
\hline Ambika & 91.31 & 23.66 & 8.00 & - & 15.33 & 2.00 & 24.33 & 10.33 & - & 83.65 \\
\hline Local variety & 88.99 & 12.00 & 6.66 & 3.00 & 3.66 & 17.00 & 23.00 & 26.33 & 6.00 & 97.65 \\
\hline \multicolumn{2}{|c|}{ Total mycoflora } & 115.64 & 57.64 & 15.65 & 45.31 & 104 & 131.98 & 63.64 & 29.32 & \\
\hline
\end{tabular}

Table.3 (b) Categorization of seeds and seedlings of pea varieties by roll paper towel method

\begin{tabular}{|c|c|c|c|c|c|c|c|c|c|c|c|c|c|}
\hline \multirow[t]{2}{*}{ Varieties } & \multicolumn{3}{|c|}{$\begin{array}{l}\text { Normal } \\
\text { Seedling }\end{array}$} & \multirow[t]{2}{*}{$\begin{array}{l}\text { Total } \\
\text { (A) }\end{array}$} & \multicolumn{3}{|c|}{ Abnormal seedling } & \multirow[t]{2}{*}{$\begin{array}{l}\text { Total } \\
\text { (B) }\end{array}$} & \multicolumn{3}{|c|}{ Ungerminated seed } & \multirow[t]{2}{*}{$\begin{array}{l}\text { Total } \\
\text { (C) }\end{array}$} & \multirow{2}{*}{$\begin{array}{l}\text { Total } \\
\text { Seedling } \\
(\mathbf{A}+\mathbf{B})\end{array}$} \\
\hline & 芯 & 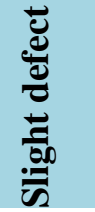 & 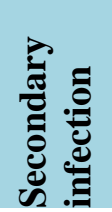 & & 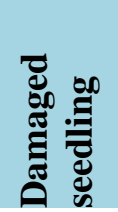 & 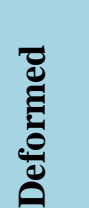 & 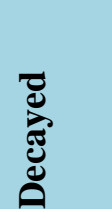 & & 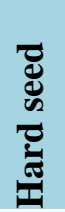 & 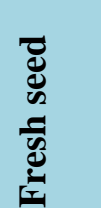 & 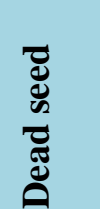 & & \\
\hline IPFD 10-12 & 2.66 & 7.66 & 76.00 & 86.32 & - & 3.33 & 4.33 & 7.66 & - & 5.00 & 1.00 & 6.00 & 93.98 \\
\hline Paras & 6.33 & 2.66 & 72.00 & 80.99 & 3.66 & 4.00 & 8.33 & 15.99 & - & - & 3.66 & 3.66 & 96.98 \\
\hline KPMR 400 & 2.33 & 7.00 & 66.33 & 75.66 & 2.66 & 3.33 & 11.00 & 16.99 & - & 3.33 & 4.00 & 7.33 & 92.65 \\
\hline Indira matar & 15.66 & - & 70.00 & 85.66 & - & 2.33 & 6.33 & 8.66 & - & 4.33 & 1.33 & 5.66 & 94.32 \\
\hline Shubhra & 10.66 & 2.33 & 61.00 & 73.99 & 3.00 & 5.66 & 16.33 & 24.99 & - & - & 0.66 & 0.66 & 98.98 \\
\hline Ambika & 7.66 & 2.66 & 67.00 & 77.32 & - & 6.33 & 7.66 & 13.99 & - & 4.00 & 3.66 & 7.66 & 91.31 \\
\hline Local variety & - & 2.33 & 47.33 & 49.66 & - & 2.33 & 37.00 & 39.33 & - & 7.00 & 2.66 & 9.66 & 88.99 \\
\hline
\end{tabular}


Table.4 Detection of mycoflora associated with pea seeds by deep freeze method

\begin{tabular}{|c|c|c|c|c|c|c|c|}
\hline \multirow[t]{2}{*}{ Varieties } & \multirow{2}{*}{$\begin{array}{l}\text { Germination } \\
(\%)\end{array}$} & \multicolumn{5}{|c|}{ Frequency of mycoflora associated (\%) } & \multirow{2}{*}{$\begin{array}{l}\text { Total } \\
\text { Frequency } \\
(\%)\end{array}$} \\
\hline & & $\dot{\square}$ & 离 & 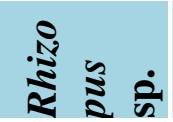 & 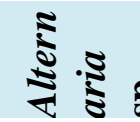 & 胥胥 & \\
\hline IPFD 10-12 & 88.75 & 34.50 & 13.75 & 37.25 & - & -2 & 85.50 \\
\hline Paras & 93.50 & 10.00 & 16.50 & 25.75 & 8.25 & 3.50 & 64.00 \\
\hline KPMR 400 & 76.25 & 25.75 & - & 41.25 & 37.50 & - & 104.50 \\
\hline $\begin{array}{l}\text { Indira } \\
\text { matar }\end{array}$ & 89.50 & 16.00 & 11.25 & 38.50 & - & - & 65.75 \\
\hline Shubhra & 97.75 & 13.50 & 6.25 & 25.50 & - & - & 45.25 \\
\hline Ambika & 86.50 & 31.25 & 8.75 & 46.00 & 6.75 & - & 92.75 \\
\hline $\begin{array}{l}\text { Local } \\
\text { variety }\end{array}$ & 55.25 & 35.75 & 30.00 & 37.50 & 7.50 & 31.00 & 141.75 \\
\hline \multicolumn{2}{|c|}{ Total mvcoflora } & 166.75 & 86.50 & 251.75 & 60.00 & 34.50 & \\
\hline
\end{tabular}

As compare to other mycoflora, Aspergillus flavus was most frequently associated mycoflora with pea varieties and Basidiophora sp. were least frequent in all pea varieties. Alternaria sp., Curvularia sp., Chaetomium sp. and Penicillium sp. were not detected in this method, which were detected in standard blotter paper method.

Grzelak (1973), Ali (1982), Nagerabi et al., (2000), Begum et al., (2004), Verma et al., (2004) and Ozgonen et al., (2011) also detected the seed borne mycoflora associated with pea seeds by agar plate method. Patil et al., (2012), Pradhan (2014) and Chaudhary et al., (2017) in pigeopea; Dawar et al., (2007), Shaker et al., (2010), Sontakke et al., (2014), Mohana et al., (2015), Trivedi et al., (2015), Kumar (2016), Hirwani (2016) in chickpea and Rathod et al., (2012) in various legumes screened the mycoflora by this method. Present study is in corroboration with the findings of above researcher.

\section{Roll paper towel method}

As per ISTA, seeds get germinated to form seedlings, categorised as normal seedlings and abnormal seedlings. Normal seedlings include intact seedlings, seedlings with slight defect and seedlings with secondary infection; Abnormal seedlings include damaged, deformed and decayed seedlings. Whereas, ungerminated seeds include hard seeds, fresh seeds and dead seeds.

Seed lot of seven varieties of pea were examined for associated seed borne mycoflora in varying frequencies with normal seedling, abnormal seedling and ungerminated seeds by roll paper towel method. It was observed that presence of mycoflora may be the cause of abnormalities and failure in germination. In this method mycoflora were found associated with seeds and seedling of pea varieties. Maximum frequency of mycoflora were recorded [Table 3(a) and (b)] from local variety seed lot $(97.65 \%)$ and mycoflora detected were Aspergillus flavus (12\%), Aspergillus niger (6.66\%), Aspergillus fumigatus (3\%), Trichoderma sp. (3.66\%), Alternaria sp.(17\%), Curvularia sp. (23\%), Chaetomium sp. (26.33\%) and Rhizopus sp. $(6 \%)$ and it showed least germination (88.99\%) with normal seedling (49.66\%) and abnormal seedling $(39.33 \%)$ followed by frequency of mycoflora in Ambika (83.65\%), KPMR 400 (82.97\%), IPFD 10-12 (79.3\%), 
Indira matar (78.31\%) and Paras (74.65\%) and germination percentage recorded in all these pea varieties were 91.31, 92.65, 93.98, 94.32 and 96.98, respectively. Shubhra variety showed minimum frequency of mycoflora (66.65\%) includes Aspergillus flavus (17.33\%), Aspergillus niger (3.66\%), Trichoderma sp. (7\%), Curvularia sp. (32\%), Chaetomium sp. (3.66\%), Rhizopus sp. (3\%) and it showed highest germination (98.98\%) with normal seedling (73.99\%), abnormal seedling (24.99\%) and ungerminated seeds $(0.66 \%)$.

In all varieties of pea, relative abundance of Curvularia sp. (131.98\%) were recorded and it was detected most frequently from pea varieties Shubhra (32\%), Ambika (24.33\%) and local variety (23\%) followed by Aspergillus flavus (115.64\%), Alternaria sp. (104\%), Chaetomium sp. (63.64\%), Aspergillus niger (57.64\%), Trichoderma sp. (45.31\%) and Rhizopus sp. (29.32\%). Aspergillus fumigatus (15.65\%) were least frequent mycoflora recorded in pea varieties in roll paper towel method. Commonly occurring seed borne mycoflora found associated with seeds of chickpea and pigeonpea were reported by Singh et al., (2014) and seed borne mycoflora of pigeonpea by Pradhan (2014) were in conformity with the findings of present study in which most common fungi in varying frequencies and their impact on germination were recorded.

\section{Deep freeze method}

Seed lots of pea varieties were evaluated for the associated seed borne mycoflora by using deep freeze method and data presented in table 4. Frequency of mycoflora associated were maximum in local variety $(141.75 \%)$ and mycoflora detected as Aspergillus flavus (35.75\%), Aspergillus niger (30\%), Rhizopus sp. (37.5\%), Alternaria sp. (7.5\%) and
Curvularia sp. (31\%) with lowest germination (55.25\%). It was followed by KPMR 400 (104.5\%), Ambika (92.75\%), IPFD10-12 $(85.5 \%)$, Paras $(64 \%)$ and Indira matar $(65.75 \%)$ with the germination percentage 76.25, 86.5, 88.75, 93.5 and 89.5, respectively. Least frequency of mycoflora recorded from Shubhra variety $(45.25 \%)$ and mycoflora detected as Aspergillus flavus (13.5\%), Aspergillus niger (6.25\%), Rhizopus sp. $(25.5 \%)$ and highest germination $(97.75 \%)$ was also recorded.

In this method, relative abundance of Rhizopus sp. (251.75\%) was recorded, followed by Aspergillus flavus (166.75\%), Aspergillus niger (86.5\%) and Alternaria sp. (60\%). Frequency of Rhizopus sp. were found maximum in Ambika variety (46\%), followed by KPMR 400 (41.25\%) and Indira matar $(38.5 \%)$. Frequency of occurrence was least in Curvularia sp. (34.5\%) and it was present only in local variety (31\%) and Paras (3.5\%) variety.

In this method mycoflora associated with seeds of pea varieties were observed maximum in local variety with lower germination percentage, while minimum frequency of mycoflora were observed from seeds of Shubhra variety with higher germination than the other varieties taken in the study. Dawar et al., (2007) also detected seed borne mycoflora in chickpea seed by deep freeze method.

\section{References}

Ali, S.M., Paterson, J., Crosby, J. 1982. A standard technique for detecting seedborne pathogens in peas, chemical control and testing commercial seed in South Australia. Australian journal of experimental agriculture and animal husbandry. 22(117): 348-352.

Anonymous. 1966. International Seed Testing 
Association. International rules for seed testing. Proc. Int. Seed Test. Assoc. 31: $1-152$.

Ashwini, C. and Giri, G.K. 2014. Detection and transmission of seed borne mycoflora in green gram and effect of different fungicides. Int. J. Adv. Res. 2(5): 1182-1186.

Barnett, H.L. 1962. Illustrated genera of imperfect fungi. $2^{\text {nd }}$ ed. Burgess Publishing Company, Minn.

Barua, J., M.M., Hossain, I., Hossain, A.A.M., Syedur, R. and Sahel, M.A.T. 2007. Control of Mycoflora of farmer stored seed of mung bean. Asian J. Plant Science. 6(1): 115-121.

Begum, M.M., Sariah, M., Puteh, A.B. and Abidin, M.A.Z. 2008. Pathogenicity of Colletotricum truncatum and its influence on soybean seed quality. Int. J. Agric. Biol. 10: 393- 398.

Chaudhary, A., Sharma, H., Jehani, M. and Sharma, J.K. 2017. Seed Mycoflora Associated with Pigeonpea (Cajanus cajan (L.) Millsp.), their significance and the Management. Journal of pure and applied microbiology. 11(1): 567575.

Dawar, S., Syed, F. and Ghaffar, A. 2007. Seed borne fungi associated with chickpea in Pakistan. Pak. J. Bot. 39(2): 637-643.

Diaz, C., Hossain, M., Bose, M.L., Mercea, S. and Mew, T.W. 1998. Seed quality and effect on Rice yield: finding from farmers participatory experiment in Central Luzon, Philippines. J. Crop. Sci. 23(2): 111-119.

Ellis, M.B. 1976. More dematiaceous hypomycetes. CABI International, Wallingford, UK.

Geetanjali, K., Giri, G.K. and Patil, A.N. 2014. Detection of seed borne fungi in mungbean from rain affected seed. J. Pl. Dis. Sci. 9(1): 91-93.

Ghangaokar, N. M. and Kshirsagar, A.D.
2013. Study of seed borne fungi of different legumes. An Int. Peerreviewed J. 2(1):32-35.

Grzelak, K. and Illakowicz, A. 1973. Fungi of the genus Ascochyta in laboratory testing of seed health of pea (Pisum sativum L.). 3/4: 155-161.

Hirwani, S. 2016. Studies on mycoflora derived seed leachate as an indicator of seed health in pulses. M. Sc. (Ag.) Thesis submitted to Indira Gandhi Krishi Vishwavidyalaya, Raipur (C.G.).

I.S.T.A. 1976. Seed health testing. International rules for seed testing. Seed Sci. Technol. 4: 31-34.

ICRISAT. 1978. A pictorial guide to the identification of seed borne fungi of sorghum, pearl millet, chickpea, pigeonpea and groundnut. International crops research institute for the semi-arid tropics. Info Bulletin. 34

Khan, A.A., Lubna, S., Kawser, J., Mian, I.H., Akanda, M.A.M. 2006. Effect of physical condition of pea seed on prevalence of seed-borne fungi, germination and seedling vigour. Bangladesh J. Pl. Pathol. 22(1/2): 8589.

Kumar, N. 2016. Food Seed Health of Chick Pea (Cicer arietinum L.) at Panchgaon, Gurgaon, India. Adv. Crop. Sci. Tech. $4: 4$.

Limonard, T. 1968. A modified blotter test for seed health. Netherlands J. Pl. Path. 72: 319-321.

Margaret, E., Neeraja, P.V and Rajeswari, B. 2013. Screening of Seed Borne Mycoflora of Cicer arietinum L. Int. J. Curr. Microbiol. App. Sci. 2(8): 124130.

Mohana, D.C., Thippeswamy, S., Abhishek, R.U., Shobha, B. and Mamatha, M.G. 2015. Studies on seed-borne mycoflora and aflatoxin B1 contaminations in food based seed samples: Molecular detection of mycotoxigenic Aspergillus 
flavus and their management. International Food Research Journal. 23(6): 2689-2694.

Muskett, A.K. and Malone, J.P. 1941. The alternate method for the examination of flax seed for the presence of seed borne parasites. Ann. Appl. Biol. 28: 8-13.

Nagerabi, S.A.F., Shafie, A.E. and Abdalla, A.H. 2000. Composition of mycoflora and aflatoxins in pea seeds from the Sudan. Kuwait journal of science and engineering. 27(1):109-122.

Narayan, M.G. and Ayodhya, D.K. 2013. Study of seed borne fungi of different legumes. J. Trends in Life Sci. 2(1): 3235.

Neergaard, P. 1977. Seed Pathology. Vol-I and Vol-II, The Macmillan Press Ltd., London.

Ozgonen, H. and Gulcu, M. 2011. Determination of mycoflora of pea (Pisum sativum) seeds and the effects of Rhizobium leguminosorum on fungal pathogens of peas. African Journal of Biotechnology. 10(33): 6235-6240.

Patil, D.P., Pawar, P.V. and Muley, S.M. 2012. Mycoflora associated with Pigeonpea and Chickpea. Int. Multidisciplinary Res. J. 2(6): 10-12.

Pradhan, A. 2014. Studies on seed mycoflora associated with pigeonpea (Cajanus cajan (L.) Millsp). M.Sc. (Ag.) Thesis submitted to Indira Gandhi Krishi Vishwavidyalaya, Raipur (C.G.).

Pradhan, S. 2017. Seed health evaluation of mungbean (Vigna radiata (L.) Wilczek) grown in agro-climatic zones of Chhattisgarh. M. Sc. (Ag.) Thesis submitted to Indira Gandhi Krishi Vishwavidyalaya, Raipur (C.G.).

Rathod, L.R., Jadhav, M.D., Mane, S.K., Muley, S.M. and Deshmukh, P.S. 2012. Seed borne Mycoflora of legume seeds. Int. J. Adv. Biotech. Res. 3(1): 530-532.

Rauf, B.A. 2000. Seed-borne disease problems of legume crops in Pakistan. Pakistan J. Sci. Indu. Res. 43(4): 249254.

Shaker, M., Momin, R. K. and Hashmi, S. 2010. Isolation and identification of some pulses Mycoflora. Bionano frontier. 3(2): 321-324.

Singh, S., Sinha, A. and Mishra J. 2014. Evaluation of different treatment on the occurrences of seed borne fungi of mungbean Vigna radiata (L.) Wilczek seed. Academic Journals. 9(44): 33003304.

Sontakke, N.R. and Hedawoo, G.B. 2014. Mycoflora associated with seeds of chickpea. Int. J. of Life Sciences. A2: 27-30.

Trivedi, L. and Rathi, Y.P.S. 2015. Detection of seed mycoflora from chickpea wilt complex seed borne Fusarium oxysporum f.sp. ciceri diseased seeds. World Journal of Pharmacy and Pharmaceutical Sciences. 44: 12421249.

Verma, S., Dohroo, N.P. 2004. Seed mycoflora of Pisum sativum in Himanchal Pradesh. Plant disease research Ludhiana. 19(2): 189.

Yaklich, R.W. 1985. Rules for testing seeds. Journals of seed technology. 6(2).

\section{How to cite this article:}

Ashruti Kesharwani, N. Lakpale, N. Khare and Tiwari, P.K. 2018. Seed Health Evaluation of Pea Varieties by Incubation Methods. Int.J.Curr.Microbiol.App.Sci. 7(08): 601-611. doi: https://doi.org/10.20546/ijcmas.2018.708.065 